\title{
Still's-like Disease Induced by Breast Implants in a Middle-Aged Female Health Professional
}

\author{
Giagkos Lavranos, Demetra Kouma, Antonis Deveros, Georgia Pigiotou, Georgios Samanis, Chrysostomos Zintilis \\ Department of Internal Medicine, Nicosia University General Hospital, Nicosia, Cyprus
}

Received: 03/10/2016

Accepted: $24 / 10 / 2016$

Published: 04/11/2016

How to cite this article: Lavranos G, Kouma D, Deveros A, Pigiotou G, Samanis G, Zintilis C. Still's like disease induced by breast implants in a middle-aged female health professional. EJCRIM 2016;3: doi:10.12890/2016_000513.

Conflicts of Interests: The Authors declare that there are no competing interests.

This article is licensed under a Commons Attribution Non-Commercial 4.0 License

\section{ABSTRACT}

We report a case of silicone-induced Still's-like inflammatory syndrome diagnosed after extensive investigation in the department of internal medicine of a secondary hospital. A 45-year-old female healthcare specialist presented with fever, headache, sore throat, myalgia and fatigue. A history of breast augmentation surgery in 2007 was confirmed. Physical examination of the patient was normal while laboratory tests showed a very high white blood cell count. The patient was admitted to hospital due to suspected infection and further laboratory tests were carried out. Inflammatory markers were persistently high despite empirical treatment with doxycycline for possible infection by Rickettsia/Coxiella. As the fever did not resolve and the patient had not improved after more than 3 weeks, the case was investigated as fever of unknown origin. Eventually, the existence of liver granulomas as well as the presence of anti-silicone antibodies confirmed the diagnosis of silicone-induced Still's-like inflammatory syndrome. In conclusion, this case supports a linkage between silicone breast implants and autoimmune connective tissue diseases.

\section{LEARNING POINTS}

- Consider and investigate a silicone-induced foreign body reaction as a cause of fever of unknown origin.

- Consider the possible harmful outcomes of breast augmentation.

- Develop a greater understanding of the potential linkage between silicone breast implants and autoimmune connective tissue diseases.

\section{KEYWORDS}

Breast implants, fever of unknown origin, silicone, Still's disease

\section{INTRODUCTION}

Despite the extensive use of breast implants for cosmetic surgery, a relatively limited number of publications have explored their potential side effects ${ }^{[1]}$, including mimicking autoimmune conditions such as Still's disease ${ }^{[2]}$. Nevertheless, the availability of diagnostic tests to measure anti-silicone antibodies in the serum ${ }^{[1,3]}$ may help confirm their potential causative role in atypical presentations of autoimmune disorders, despite the fact that this pathophysiological association remains controversial ${ }^{[4]}$. 


\section{CASE REPORT}

A 45 -year-old woman reported fever (up to $39^{\circ} \mathrm{C}$ ) with rigor over the past 2 weeks. She also had a headache, sore throat, tenderness and pain in the anterior neck, non-productive cough, myalgia, arthralgia and fatigue. She was a nurse in a general secondary hospital and reported that she had undergone breast augmentation with breast implants for cosmetic reasons in 2007.

Physical examination performed on admission revealed minimal findings. Cardiac examination showed the sinus rhythm was regular and no additional heart sounds were observed. Respiratory examination showed normal breath sounds in both lungs. The abdomen was soft and non-tender with normal bowel sounds and no organomegaly or lympadenopathy was present. The skin was unremarkable and there were no major findings on neurological and sensory examination.

A simple chest $\mathrm{x}$-ray and ECG were also normal and the patient was admitted for further investigation. The laboratory tests on admission showed WBC 12,300 (N 85.6\%, L 6.6\%, M 7\%, E 0.6\%), Hgb 10.9 g/dl, MCV 76 and PLT 300,000. Urea, creatinine, electrolytes and MSU were normal; ALT 151, AST 97.

As the differential diagnosis included rickettsial infection, leptospirosis and viral infection, the initial investigation included testing for WeilFelix reaction and semi-quantitative ELISA for Rickettsia typhi, Leptospira and Coxiella burnetii antibodies to confirm rickettsial infection. Extended viral serology for acute infection was also performed.

The patient was provided with empirical treatment (doxycycline $100 \mathrm{mg}$ twice a day). However, fever had not responded to doxycycline after 5 days and inflammatory markers also persistently increased: hs-CRP 8.86, ESR 34, PCT 0.23.

Further investigation with blood culture sets for aerobic and anaerobic bacteria failed to detect any pathogenic bacteria. Serology revealed negative Coxiella phase I and II, R. typhi, Leptospira and Weil-Felix reaction, and negative blood cultures and extended viral screening. Neither Legionella pneumophila serogroup 1 antigen nor Streptococcus pneumoniae antigen in urine was detected.

After 3 weeks of a temperature of over $38.3^{\circ} \mathrm{C}$ and no diagnosis after 1 week of investigation, the fever was officially defined as fever of unknown origin.

An extensive investigation plan included the following tests with no findings:

- $\quad$ Autoimmunity serological screening, antithyroid antibodies

- Ultrasound of the neck, thyroid gland and abdomen

- $\quad$ Laryngoscopy, otoscopy: unremarkable findings

- $\quad$ TST and bronchoscopy (BAL, b Koch, cytology)

- LP: normal

- $\quad$ Ophthalmological examination, fundoscopy: no major findings

- $\quad$ Breast mammography

- Gastroscopy, biopsy and gastric fluid for b Koch

- Colonoscopy: normal

- Bone marrow biopsy

- Protein electrophoresis

- Whole body gallium scan

The results of a Tru-Cut liver biopsy were still pending at this point.

A follow-up clinical examination on day 24 revealed pain, swelling, redness of the left ankle and a salmon-pink rash over the trunk and lower extremities as well as dyspnoea with fine crackles and signs of pericarditis.

A CT of the chest (Fig. 1) revealed pericardial fluid $1 \mathrm{~cm}$ in diameter and mediastinum nodules $11 \mathrm{~mm}$ in diameter, while a transthoracic echo (Fig. 2) confirmed a pericardial effusion $0.5 \mathrm{~cm}$ in diameter. Serum ferritin was also increased (5,400).

These data suggested a new possible differential diagnosis, Still's disease. The patient was placed on prednisone and colchicine with rapid fever regression. The results of the liver biopsy performed a week previously (Fig. 3) revealed chronic hepatitis and sclerotic-hyaline nodules secondary to foreign body exposure. Subsequently, anti-silicone antibody testing ordered via a reference laboratory in the UK was positive ${ }^{[1]}$. Therefore, the final diagnosis was silicone-induced Still's-like inflammatory syndrome.

The treatment plan included $40 \mathrm{mg}$ of prednisone daily, colchicine and removal of the breast implants within a month. 
The patient experienced rapid symptom regression under corticosteroid treatment, which persisted after steroid discontinuation. The patient is symptom-free without any medication 3 years later.
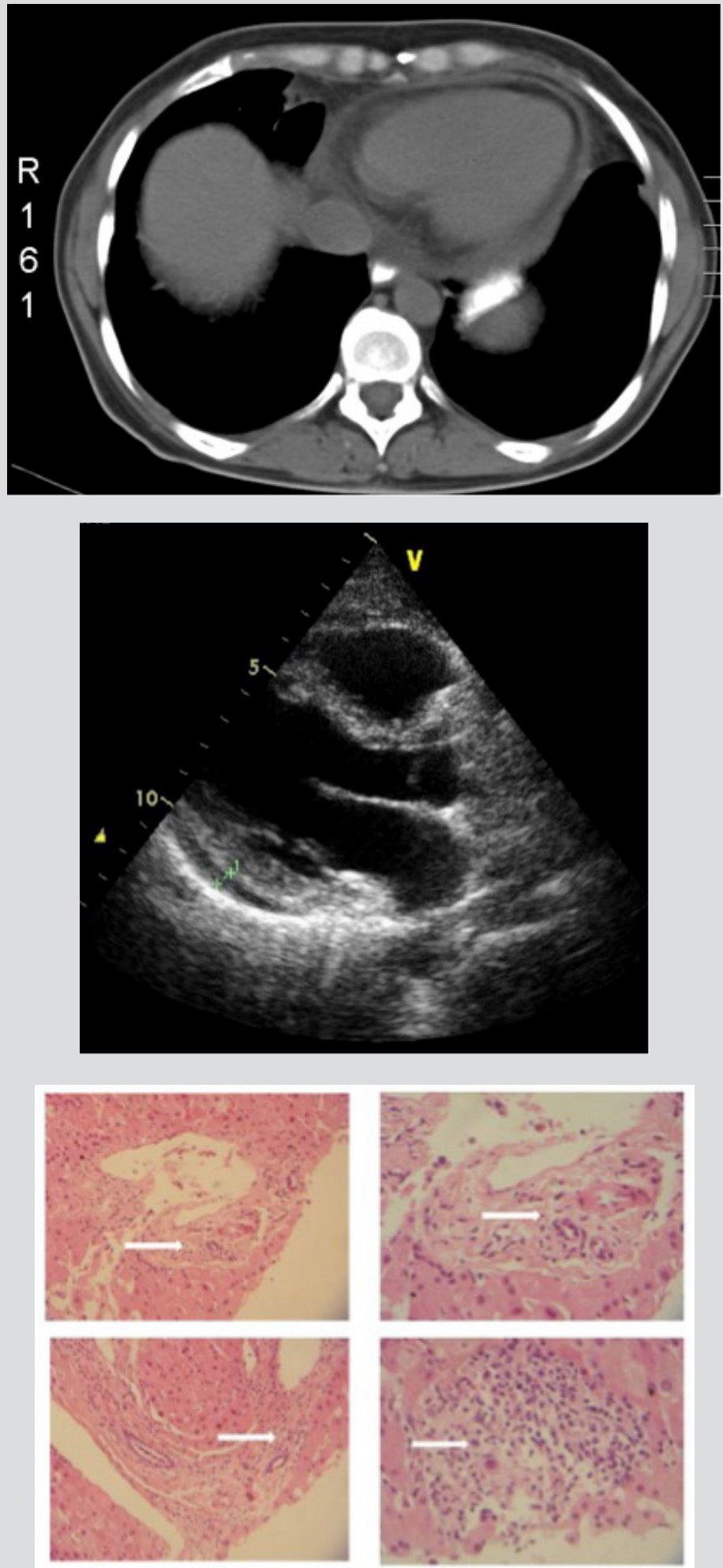

Figure 1. CT chest showing pericardial fluid $1 \mathrm{~cm}$ in diameter and mediastinum nodules $11 \mathrm{~mm}$ in diameter

Figure 2. Echo showing pericardial effusion $0.5 \mathrm{~cm}$ in diameter

Figure 3. Core biopsy of the liver (segmental staining, H\&E) demonstrating chronic hepatitis and sclerotic-hyaline nodules secondary to foreign body exposure (silicone-induced hepatotoxicity). Top left: Increase in collagenous/fibrous stroma with areas of hyalinization (100x). Top right: $200 \times$ Magnification of top left image, showing extensive hyalinization of pericholangiolar stroma. Bottom left: Portal triad and central vein segment of hepatic lobule with extensive inflammatory infiltration (100x). Bottom right: $200 \times$ Magnified view of pericholangiolar area showing inflammatory infiltration by lymphocytes, plasma cells and few eosinophilic leucocytes, indicating possible foreign body granuloma 


\section{DISCUSSION}

The endemic nature of Rickettsia spp. and C. burnetii infection has been well documented in Cyprus, with 20-25 cases reported via the WHO compulsory notification system annually, which corresponds to an incidence rate of about 2.5 new cases per 100,000 inhabitants annually. Diagnostic confirmation may be provided via serological or molecular testing, with the latter technique offering higher specificity and sensitivity but being much more expensive. Despite the significant impact of rickettsial diseases in terms of public health, limited data are available on their clinical spectrum or their responsiveness to initial doxycycline therapy (standard of care) which therefore should only be offered following laboratory confirmation or strong epidemiological and clinical suspicion of the condition.

The Yamaguchi criteria for diagnosing Still's disease require the presence of five features, with at least two being major diagnostic criteria ${ }^{[2]}$. Our patient presented with major fever of at least $39^{\circ} \mathrm{C}$ lasting at least 1 week, arthralgias or arthritis lasting 2 weeks or longer, a nonpruritic salmon-coloured macular or maculo-papular skin rash usually found on the trunk or extremities during febrile episodes, leukocytosis $(10,000 / \mu l$ or greater) with at least $80 \%$ granulocytes, and other minor criteria (sore throat, abnormal liver function studies, particularly elevations in aspartate and alanine aminotransferase and lactate dehydrogenase concentrations, and negative tests for antinuclear antibody and rheumatoid factor). The patient did not show any Yamaguchi exclusion criteria ${ }^{[2]}$ (the presence of any infection, malignancy or other rheumatic disorder known to mimic the clinical features of Still's disease).

Based on the laboratory findings, the presence of anti-silicone antibodies and foreign body granulomas in the liver confirms that an inflammatory reaction was induced by silicone and therefore silicone breast implants were the causative agent of Still-like inflammatory syndrome ${ }^{[3]}$. Although previous research found no definite evidence to support an association between silicone breast implants and autoimmune connective tissue diseases ${ }^{[4]}$, many cases indicate a potential linkage between them ${ }^{[5-9]}$. In recent years, the term autoimmune/ inflammatory syndrome induced by adjuvants (ASIA) has been proposed for autoimmune conditions either primarily diagnosed or reactivated after prosthetic material has been placed in or has ruptured in the patient's body $\mathrm{y}^{[5]}$.

It is not clear whether breast implants should be removed in cases of new onset or re-activation of autoimmune conditions such as Still's disease $^{[6,7]}$. Current recommendations indicate that the evidence supports routine remova[ ${ }^{[4]}$. In our case, the existence of both anti-silicone antibodies and foreign body granulomas in the liver were considered sufficient to suggest implant removal, resulting in disease regression. Nevertheless, other authors have reported cases where short-term steroid treatment has resulted in symptom regression without the need for implant removal ${ }^{[8]}$ as well as cases where the patient remained steroid dependent even after remova ${ }^{[9]}$.

In conclusion, further investigation should be considered in order to develop a deeper understanding of the possible correlation between silicone breast implants and autoimmune connective tissue diseases.

\section{REFERENCES}

1. Vojdani A, Brautbar N, Campbell AW. Antibody to silicone and native macromolecules in women with silicone breast implants. Immunopharmacol Immunotoxicol 1994;16:497523.

2. Yamaguchi M, Ohta A, Tsunematsu T, Kasukawa R, Mizushima Y, Kashiwagi H, et al. Preliminary criteria for classification of adult Still's disease. J Rheumatol 1992;19:424-430.

3. Oliver DW. Anti-silicone antibodies and silicone containing breast implants. Br J Plast Surg 2000;53:410-414.

4. Tugwell P, Wells G, Peterson J, Welch V, Page J, Davison C, et al. Do silicone breast implants cause rheumatologic disorders? A systematic review for a court-appointed national science panel. Arthritis Rheum 2001;44:2477-2484.

5. Jara LJ, Medina G, Gómez-Bañuelos E, Saavedra MA, Vera-Lastra O. Still's disease, lupus-like syndrome, and silicone breast implants. A case of 'ASIA' (Shoenfeld's syndrome). Lupus 2012;21:140-145.

6. Błasiak A, Błachowicz A, Gietka A, Rell-Bakalarska M, Franek E. Still's disease in patient with silicone breast implants: case report. Pol Arch Med Wewn 2008;118:65-67.

7. Katayama I, Umeda T, Nishioka K. Adult Still's-disease-like illness in a patient with silicone breast implants. Clin Rheumatol 1998;17:81-82.

8. Montano M, Vaatola M, Santoro L, La Regina M, Curigliano V, Manna R, et al. Systemic inflammatory diseases and silicone breast prostheses: report of a case of adult still disease and review of the literature. Am J Med Sci 2004;327:102-104.

9. Cretel E, Richard MA, Jean R, Durand JM. Still's-like disease, breast prosthesis and collagen implants. Rheumatol Int 2001;20:129-131. 